\title{
Study of functional outcome in low grade spondylolisthesis operated by pedicular screw instrumentation and fusion
}

\author{
C. Panchal L. ${ }^{1}$, L Sarukte V. ${ }^{2 *}{ }^{*}$ N Bhanushali R. ${ }^{3}$ \\ DOI: https://doi.org/10.17511/ijmrr.2021.i01.05 \\ 1 Lalit C. Panchal, Honorary Professor, K.B. Bhaba Hospital, Mumbai, Maharashtra, India. \\ 2* Vijay L Sarukte, Additional Associate Professor, K.B. Bhaba Hospital, Mumbai, Maharashtra, India. \\ ${ }^{3}$ Ravi N Bhanushali, (DNB), Spine Specialist, K.B. Bhaba Hospital, Mumbai, Maharashtra, India.
}

Background: Functional outcome following instrumental spinal surgery for spondylolisthesis in physically energetic patients is crucial. The present study was undertaken to evaluate the functional outcome of low-grade spondylolisthesis accompanied by low back pain with or without radiculopathy, with standard surgical procedure posterior lumbar interbody fusion with instrumentation. Method: In this study total of 40 patients were operated on for low-grade spondylolisthesis by posterior stabilization using a pedicular screw rod system and posterior lumbar interbody fusion. All the patients were followed up till 6 months after surgery and functional outcomes were noted. Results: Assessment of this series it was observed that, $57.5 \%$ of the patient had excellent outcome, $37.5 \%$ had a good outcome and $97.5 \%$ of the study population had satisfactory outcome (improvement in clinical results). There was a significant improvement in pain intensity, walking, lifting, standing, sleeping after surgery. The mean ODI difference between preoperative and post-operative at 6 months follow up was $36.12 \%$ (16.75). In the outcome, $62.5 \%$ of the patient consisted of severe disability and $32.5 \%$ were with moderate disability (total-95\%) while postoperative $87.5 \%$ were with a minimal disability and only $2.5 \%$ of the study population had worsened i.e crippled. Conclusion: The study concluded that surgery in form of decompression with instrumentation and posterior lumbar interbody is a safe and effective method to treat spondylolisthesis.

Keywords: Spondylolisthesis, Radiculopathy, Posterior, Pedicular screw, Fusion, Functional outcome

Corresponding Author

Vijay L Sarukte, Additional Associate Professor, K.B. Bhaba Hospital, Mumbai, Maharashtra, India.

Email: vijaysarukte69@gmail.com

\section{How to Cite this Article}

Panchal LC, Sarukte VL, Bhanushali RN. Study of functional outcome in low grade spondylolisthesis operated by pedicular screw instrumentation and fusion. Int J Med Res Rev. 2021;9(1):34-40.

Available From

https://ijmrr.medresearch.in/index.php/ijmrr/article/ view/1258
To Browse

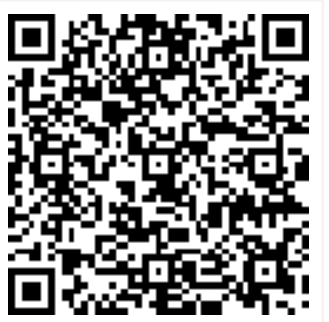

Manuscript Received 2021-01-22

Conflict of Interest No
Review Round 1 2021-02-01

Funding Nil

Review Round 2
2021-02-14
Ethical Approval
Yes

Review Round 3

Accepted 2021-02-23

Plagiarism $\mathrm{X}$-checker $7 \%$

Note

(C) 2021 by Lalit C. Panchal, Vijay L Sarukte, Ravi N Bhanushali and Published by Siddharth Health Research and Social Welfare Society. This is an Open Access article licensed under a Creative Commons Attribution 4.0 International License https://creativecommons.org/licenses/by/4.0/ unported [CC BY 4.0]. 


\section{Introduction}

Chronic low back pain (LBP) affects the physical function and quality of life of a large number of individual and one of the causes of back pain is spondylolisthesis. In this condition one vertebra (usually L5) becomes misaligned anteriorly (slips forward) concerning the vertebra below. However, the incidence of spondylolisthesis is $5-6 \%$ in white males and $2-3 \%$ in females, the most common level is a lumbosacral junction (L5-S1) in $82 \%$ and lumbar $4-5(L 4-5)$ in $11 \%$ [1]. Spondylolisthesis is classified into five types based on anatomy and aetiology: Dysplastic, Isthmic, Degenerative, traumatic, pathological and iatrogenic spondylolisthesis). This condition has to be differentiated from, spondylolysis which can be present as a defect in the pars interarticularis without slippage. Meyerding grading is used to determine the slip [2].

Various surgical techniques of spinal fusion surgery have been developed to remove pain-generating tissues and to decrease the patients' pain by stabilization of one or more motion segments. However ideal surgical treatment for spondylolisthesis remains controversial [3]. Spinal fusion with pedicle screw fixation is significantly more effective in reducing both LBP and radicular pain, providing immediate stability of the column, improving the rate of fusion, and leading to reestablishment of physiological lordosis [4]. The main question in today's spine surgery is "When to fuse", "How to fuse" and "results of fusion". The outcome in spondylolisthesis is related more to the preoperative neurological deficit, radiculopathy and aetiology of the indication for surgical stabilization.

The present study was conducted to review our experience with posterior lumbar interbody fusions with posterior pedicular screw instrumentation for the management of low-grade spondylolisthesis and to study its efficacy in terms of functional outcome through the adoption of the outcome measure, to study the complications associated with this treatment modality and compare the efficacy and complications with the available literature. To verify whether the quality of life is significantly influenced by surgery in cases of spondylolisthesis we analyzed patients using the Oswestry Disability Index (ODI), which is a widely accepted measurement method for the follow-up evaluation of specific procedures in cases of lumbar spine pathology.

\section{Materials and Methods}

The present prospective observational study was conducted in the Department of Orthopaedics at K. B. Bhabha municipal general hospital, Bandra West, Mumbai during a period from 2012 to 2017. Total 40 patients of either sex, age between 20-80 years and who diagnosed with spondylolisthesis of low-grade level (Meyerding grades I-II) and those experience low-back pain with radiculopathy, severe restriction of functional capabilities with failed conservative treatment for 3months were included in the study.

Patients of age $<20$ years and $>80$ years, with Grade-3 \& 4 spondylolisthesis, who have previously undergone spinal surgery or the presence of a psychiatric disorder and/or drug and/or alcohol abuse, patients with any other spinal pathologies and who did not have a regular follow up for a period of 6 months were excluded from the study.

A detailed history was taken and a thorough general physical, systemic and local examination as well as all routine and relevant investigation was done. $X-$ rays of lumbosacral spine $A$. P. view and lateral view with flexion and extension in lateral view and instability was checked for. MRI pelvis with both hips was done. Informed consent was taken from all the patients before giving any kind of treatment and its approval by the ethics committee.

Patients who fulfilled the inclusionary criteria were admitted to the hospital for surgical treatment from the outpatient department. The pre-operative assessment was carried out on all patients with the Oswestry Disability Index (ODI). A proforma was made for each patient and records were kept in a custom-built database. Patients were operated upon by Instrumentation with posterior lumbar interbody fusion for low-grade spondylolisthesis under general anaesthesia.

The image I show the pre-operative preparation of the patient, the posterior approach shown in Image 2 and implantation of the pedicle screw in Image 3. The surgical management was done by the same surgeon who is an expert in this surgery.

Postoperative plain X-rays of the lumbar spine were done of all patients; the site of surgical fixation appeared satisfactory, and they started ambulation with lumbosacral support on the third or fifth postoperative day. Spinal rehabilitation was organized for all cases with physiotherapy protocol. 
All patients were evaluated in the outpatient clinic regularly as follows 2 weeks, 3 weeks, 6 weeks, 12 weeks and finally at 6 months. ODI was used for assessment at 6 months again.

Statistical Analysis: The data were collected and analyzed with the help of PSPP software and Sigma plot version 12. Quantitative data was presented with the help of Mean \pm SD, Median and IQR, 'pre' and 'post' comparison among study groups was done with the help of Wilcoxon Signed Ranks Test as per results of Normality test. Qualitative data was presented with the help of the Frequency and Percentage table. P-value less than 0.05 was taken a significant level.

Fig-1: a) prone position, b) Scrubbing, Painting and Draping, c) Draping and marking of surgical site

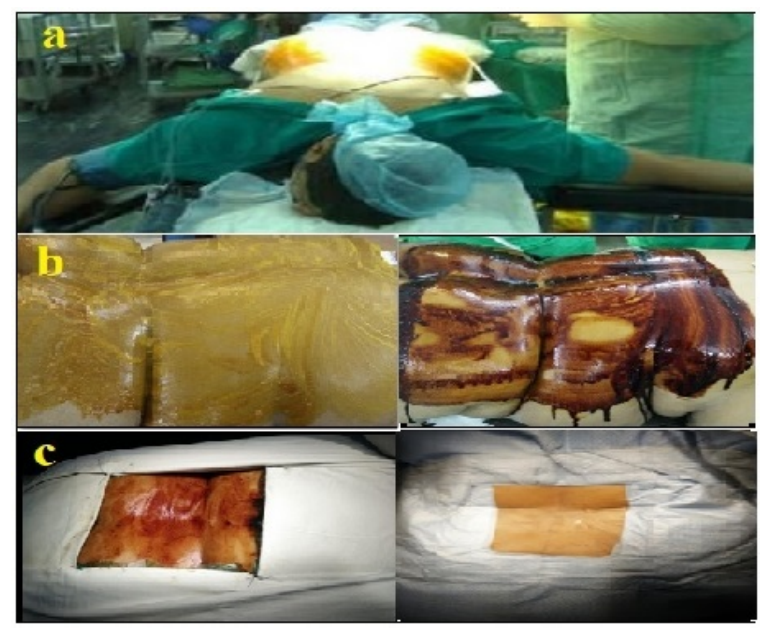

Fig-2: a) Subcutaneous Dissection with Cobb, b) complete exposure of spinous process, c) cutting spinous process with nebler, d) laminectomy with a bone rongeur, e) lamina exposed nerve root decompressed, f) lateral exposure to the transverse process

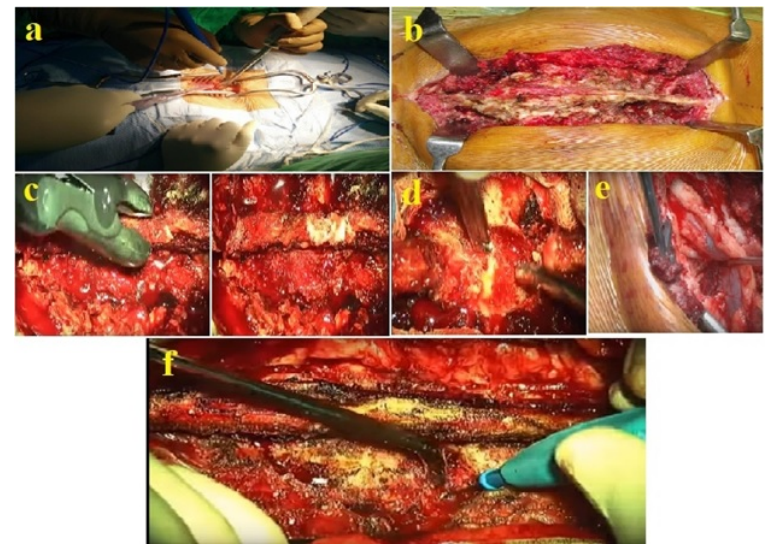

Fig-3: Implantation of pedicle screw: - a) Pedicle screw path, an entry made with an awl, length measurement with scale, tapping pedicle, screw insertion, verification under image intensifier, b) rod positioning and final rod placement, C) Closure
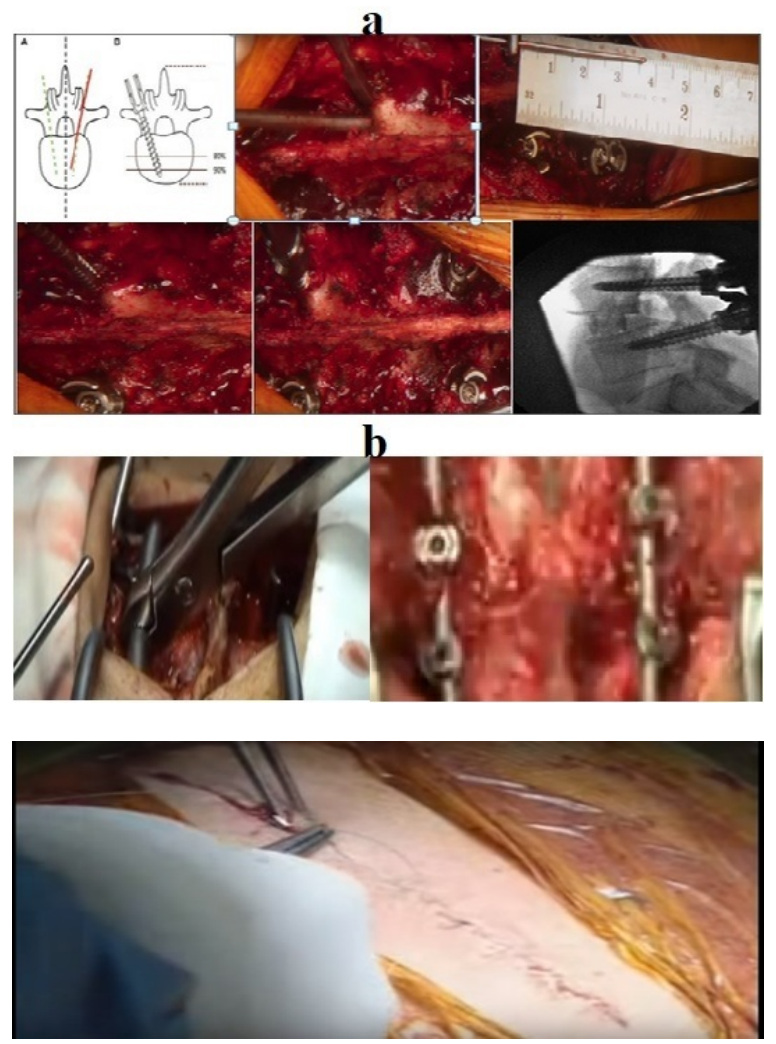

\section{Results}

A total of 40 cases of low-grade spondylolisthesis were enrolled in the study, among them, 17 $(42.50 \%)$ were male and $23(57.50 \%)$ were female. The maximum numbers of patients were in the age group of $41-60$ years (75\%) (Table 1 ) with a mean age of $55.20 \pm 7.79$ and ranged from 38-76 years. The highest level of incidence of spondylolisthesis was at L5-S1 level (57.5\%) and a maximum number of patients $(80 \%)$ had grade 1 spondylolisthesis as shown in table 1.

Table 1: Distribution of study group as per Age and diagnosis

\begin{tabular}{|l|l|l|l|}
\hline \multicolumn{2}{|c|}{ Parameters } & No. of cases & Percentage \\
\hline Age group (in years) & $31-40$ & 01 & 2.5 \\
\cline { 2 - 4 } & $41-50$ & 11 & 27.5 \\
\cline { 2 - 4 } & $51-60$ & 19 & 47.5 \\
\cline { 2 - 4 } & $61-70$ & 08 & 20 \\
\cline { 2 - 4 } & $\geq 71$ & 01 & 2.5 \\
\hline
\end{tabular}




\begin{tabular}{|l|l|l|l|}
\hline \multirow{3}{*}{ Level of spondylolisthesis } & L3 over L4 & 02 & 5 \\
\cline { 2 - 4 } & L4 over L5 & 15 & 37.5 \\
\cline { 2 - 4 } & L5 over S1 & 23 & 57.5 \\
\hline Meyedring grading & Grade I spondylolisthesis & 32 & 80 \\
\cline { 2 - 4 } & Grade II spondylolisthesis & 08 & 20 \\
\hline
\end{tabular}

The posterior approach was used in all cases under general anaesthesia. The mean operating time was approx 120 minutes with no significant intraoperative events. Blood transfusion was done in 25 patients.

Most of the patients (65\%) were mobilized by 3rd postoperative day and all patients were mobilized by 7 postoperative days. The postoperative hospital stay was maximum between 1 week in $60 \%$ of cases as shown in table 2. Regarding postoperative complications dural tear occurred in 1 patient. 1 patient developed a drop foot. Implant failure was seen in 1 patient and 1 patient (2.75\%) had evidence of early infection.

Table 2: Distribution of study group as per postoperative mobilization and hospital stay

\begin{tabular}{|c|c|c|c|}
\hline & & No. of cases & Percentage \\
\hline \multirow[t]{3}{*}{ Post-op mobilization } & 3rd post-op day & 26 & 65 \\
\hline & 5th post-op day & 12 & 30 \\
\hline & 7th post-op day & 02 & 5 \\
\hline \multirow[t]{3}{*}{ Hospital stay } & 1 week & 24 & 60 \\
\hline & $1-2$ weeks & 16 & 40 \\
\hline & Mean \pm SD (days) & \multicolumn{2}{|l|}{$406.18 \pm 1.92$} \\
\hline
\end{tabular}

After surgery, there was a significant improvement in the quality of activities of daily living including pain intensity, walking, lifting, standing, sleeping as shown in Table 3.

Table 3: Comparison of pre and postoperative parameters

\begin{tabular}{|l|l|l|l|}
\hline Parameters (Mean \pm SD) & Preoperative & Postoperative & P-value \\
\hline Pain intensity & $3.23 \pm 0.53$ & $0.28 \pm 0.50$ & 0.000 \\
\hline Lifting & $2.85 \pm 0.86$ & $0.80 \pm 0.68$ & 0.000 \\
\hline Walking & $2.28 \pm 0.50$ & $0.45 \pm 0.81$ & 0.000 \\
\hline Standing, & $2.18 \pm 0.67$ & $0.43 \pm 0.87$ & 0.000 \\
\hline
\end{tabular}

Assessment of this series it was observed that, the maximum number of patients i.e. $57.5 \%$ had excellent results and $37.5 \%$ had good results. $97.5 \%$ of the study population had a satisfactory outcome (improvement in clinical results) as depicted in figure 4.

Fig-4: Distribution of study group as per result and outcome
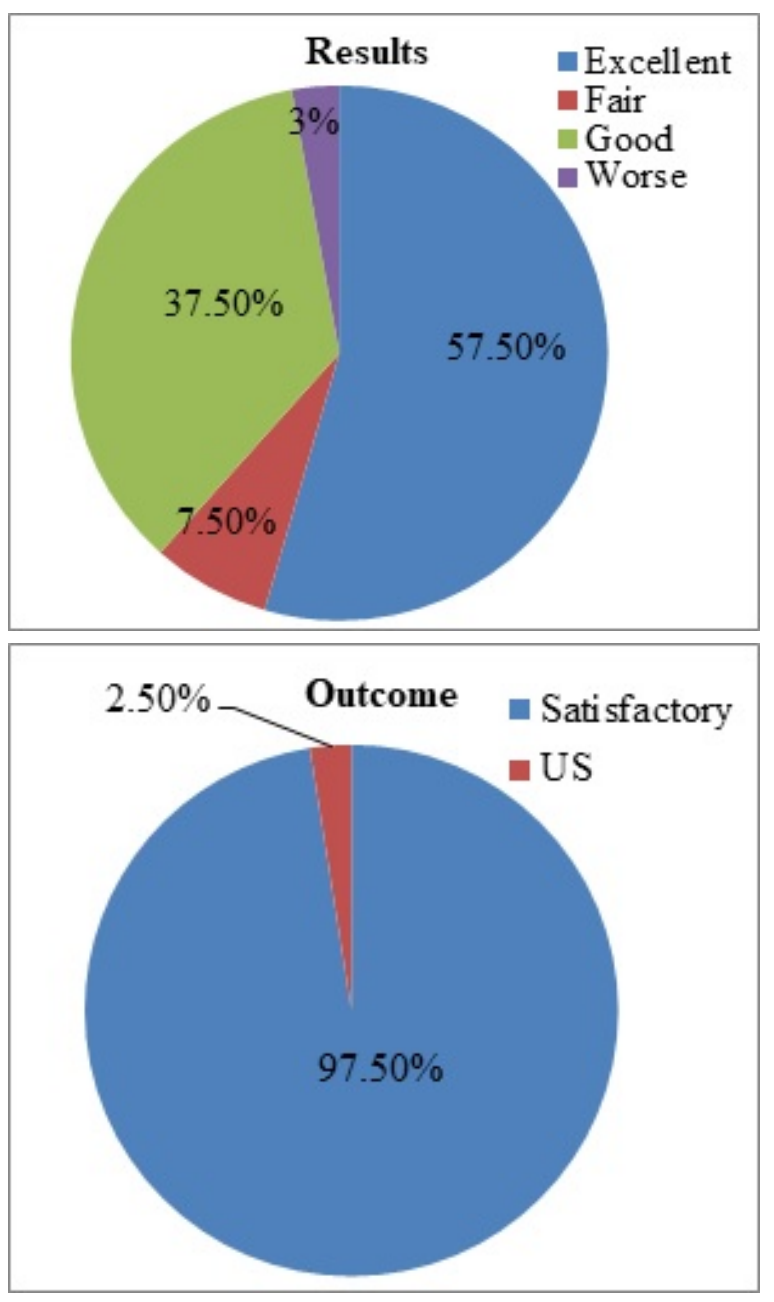

The preoperative ODI was $46.3 \%$ (22.3) and postoperative mean ODI was $10.18 \%$ (5.55) with a mean ODI difference pre and post-operative at 6 months follow up was $36.12 \%(16.75)$.

In the outcome of our study $62.5 \%$ of the patient consisted of severe disability and $32.5 \%$ were with moderate disability(total-95\%) while postoperative $87.5 \%$ were with a minimal disability and only $2.5 \%$ of the study population had worsened i.e crippled, (Table 4).

Table 4: Distribution of study group as per pre and post-operative score

\begin{tabular}{|l|l|l|l|l|}
\hline \multirow{2}{*}{ Postoperative score } & \multicolumn{3}{|c|}{ Preoperative score } & \multirow{2}{*}{ Total } \\
\cline { 2 - 4 } & Crippled & Moderate & Severe & \\
\hline Crippled & 00 & 00 & 01 & $1(2.5 \%)$ \\
\hline Minimal & 00 & 13 & 22 & $35(87.5 \%)$ \\
\hline Moderate & 02 & 00 & 02 & $4(10 \%)$ \\
\hline Total & $2(5 \%)$ & $13(32.5 \%)$ & $25(62.5 \%)$ & $40(100 \%)$ \\
\hline
\end{tabular}




\section{Discussion}

In the present study most of the patients were adults and the majority of patients belong to the age group 51-60 years. The high incidence of spondylolisthesis in this age group is due to degenerative changes in the spine. Males cases were more because males are heavy worker and female were less because of social rejection. The majority of the patients were housewives/maids (25\%-10) followed by those doing heavy manual work.

All the patients had low back pain with Radicular pain \& amp, only one with neurological deficit and were given NSAID's, bed rest, and underwent traction and physiotherapy for a variable period of 3 months. All patients have had para-spinal muscle spasm and spinal tenderness and had reduced spinal movements. Regarding the highest level of incidence was at the L5-S1 level (57.5\%), this is following the literature were the most common level is a lumbosacral junction (L5-S1) [5-7].

This is because of high Pelvis incidence which is associated with slippage and high shear forces at the lumbosacral junction, which disturbs the sagittal balance of the spine [8]. Next to L5-S1 there was L4-L5 spondylolisthesis in 15 patients i.e. $37.5 \%$ due to degenerative disease of the spine, affecting facet joints and the discs permitting forward slip nearly always at L4-L5 despite intact laminae. Lowgrade spondylolisthesis there were around $80 \%$ with grade 1 and $20 \%$ constituted grade 2 , this is comparable with the previous studies [3, 9, and 10].

Duration of symptoms was from 6 months to 7 years with a mean duration of symptoms 18 months which is similar to other studies $[11,12]$. The Indications of surgery were intractable pain not responding to conservative treatment and this was present in all the studied patients. $27.5 \%$ of cases had stenosis with spondylolisthesis and 30 cases had nerve root compression. In this 1 had stenosis with disc bulge and root compression \& 2 were detected with pars defect on MRI scan.

All patients were mobilized by 7 postoperative days and were taught post-operative protocol for 3 months. Immediate immobilization helps to reduce the chances of DVT and also in the majority of cases stable fixation in form of pedicular rod fixation with cage was done. This helped to stabilize the involved level and immediate mobilization.
About postoperative complications dural tear occurred in 1 patient and was repaired with 5/0 vicryl \& there was no CSF leakage. 1 patient developed a drop foot that was investigated by gadolinium contrast MRI and EMG-NCV, patient refused further management of foot drop. Implant failure was seen in 1 patient at 4 weeks and readmitted for it. One patient $(2.75 \%)$ had evidence of early infection. The organism isolated from the patient was Staphylococcus Aureus and infection subsided patients with a 6-week course of oral linezolid.

Advances in our understanding of patient selection, operating room environment, surgical technique and use of prophylactic antibiotics have dramatically reduced the risk of this devastating complication. Most spine infection is caused by gram-positive organisms, particularly Staph. aureus, Staph. epidermidis. Gram-negative organisms are more commonly encountered in hematogenous infections particularly those emanating from the urinary tract.

There was a significant reduction of pain intensity and other parameters like walking, lifting, standing, sleeping after surgery. This indicates that there is a significant post-surgical improvement in the quality of activities of daily living. The mean ODI difference between preoperative and post-operative at 6 months follow up was $36.12 \%$ (16.75) which was compared with other standard series [3, 11-15]. In the outcome of this study, $62.5 \%$ of the patient consisted with severe disability and $32.5 \%$ were with moderate disability (total-95\%) while postoperative $87.5 \%$ were with a minimal disability and only $2.5 \%$ of the study population had worsened i.e crippled.

These results are compared with the study done by Kaftandziev et al [16]. Regarding the subjective assessment of this series it was observed that, $57.5 \%$ of the patient had an excellent outcome and $37.5 \%$ had a good outcome \& $7.5 \%$ fair outcome and $97.5 \%$ of the study population had satisfactory outcome (improvement in clinical results). Indicating that result was statistically significant by Wilcoxon signed-rank test. This was confirmed with the previous studies conducted by Chaitanya et al [3], Mohammad et al [9] and Park et al [14].

In all cases at 6 months fusion was checked and there were no abnormal radiological findings on final follow up in all patients. Many of the patients in our series suffered from one or more systemic illness ranging from diabetes mellitus, hypertension. 
Thus spinal fusion, though a major surgery can be performed safely and effectively in patients with systemic diseases. Being a major surgery and involving procedures like bone grafting, prone position, and general anaesthesia all patients and especially those with systemic diseases should be thoroughly investigated and evaluated before anaesthesia and surgery. Diseases like rheumatoid arthritis, diabetes mellitus, hypertension etc., treated with steroids, anti-diabetic drugs and antihypertensive pre-operatively, required due care intraoperatively for meticulous handling of tissues, to avoid infection and also proper care postoperatively, to avoid complications.

In all our patients, deep vein antithrombotic prophylaxis was given with low molecular weight heparin (Rivaroxaban) for 5 days post-operatively and none of them developed DVT. In the present study spondylolisthesis indirect reduction was achieved through pedicle screws with the release of the disc and soft tissues. In addition to a wide neural decompression, a discectomy and curettage of the endplate were performed for the PLIF cage insertion which maintained the reduction and improved the neural foraminal dimensions significantly.

Moreover, the results of prospectively evaluated ODI based outcomes indicated that age and sex were not related to poorer outcome or complication in the current study. The surgery-related results indicated that outcomes were significantly improved after surgery, and these data support the need for a prospective randomized multicentre trial using various scoring system (Roland morris scale, quality of life) and radiological evaluation to determine the most appropriate method for the benefit of patients. There are several strengths of the study: 1) All types of spondylolisthesis were taken into consideration i.e isthmic and degenerative spondylolisthesis, 2) only low-grade spondylolisthesis were presented in this series which have been less studied, 3) all patients had undergone the same surgery for a single level spondylolisthesis.

\section{Conclusion}

Posterior lumbar interbody fusion and spinal decompression is an effective method in the treatment of spondylolisthesis, as it provided good spinal fusion, less complication with satisfactory clinical outcome and reduction of a slip in low grade especially.

\section{What does the study add to the existing knowledge?}

This surgery has gradually gained good popularity since its inception amongst the patient population reporting at our institute. However, this procedure provides a better outcome and with experience, proper instrumentation, proper and fusion techniques, and gives excellent results with minimal complication rate. Good outcome was mainly related to the preoperative neurological deficits. Apart from surgical management, modification of the lifestyle is also recommended to avoid failure of the surgery. Although short-term results from studies are promising, the number of patients included was relatively small and studies with larger numbers of patients are required. For the good outcome of the surgical treatment it is necessary to have a good selection of patients; there should be a clear indication for operative treatment to obtain the best results from the surgery.

\section{Author's contribution}

Dr. Lalit C. Panchal: Concept, Dr. Vijay L Sarukte: Study design, Dr. Ravi N Bhanushali: Manuscript Preparation

\section{Reference}

01. Rowe GG, Roche MB. The aetiology of the separate neural arch. J Bone Joint Surg Am. 1953;35;102-10.

[Crossref]

02. Tenny S, Gillis CC. Spondylolisthesis, [Updated 2020 Jul 21], In- StatPearls [Internet]. Treasure Island (FL)- StatPearls Publishing. 2020 Jan.

Available from: [Article] [Crossref]

03. Chaitanya M, Mittal A, Rallapalli R, Teja R, Prasad YS. (2015) Surgical Management of Spondylolisthesis by Pedicular Screw Rod System and Postero-Lateral Fusion. Open Journal of Orthopedics. 2015;5;163-174.

[Crossref]

04. Christensen FB, Hansen ES, Laursen M, Thomsen K,Bunger CE. Long-term functional outcome of pedicle screw instrumentation as support for posterolateral spinal fusion- A randomized clinical study with a 5 -year followup. Spine. 2002;27;1269-1277.

[Crossref] 
05. Shivakumar HB, Channappa TS, Singh A. Functional Outcome of Lumbo-Sacral Spondylolisthesis-Posterior Stabilisation with Moss-Miami Instrumentation and Spinal Fusion. " Journal of Evolution of Medical and Dental Sciences. 2015;4(93)15819-15822.

[Crossref]

06. Molinari RW, Sloboda JF, Arrington EC, et al. Low-grade isthmic spondylolisthesis treated with instrumented posterior lumbar interbody fusion in US servicemen. J Spinal Disord Tech. 2005;18(suppl 1)S24-9.

[Crossref]

07. Yizhar Floman, Michael A Millgram. Instrumented Slip Reduction and Fusion for Painful Unstable Isthmic Spondylolisthesis in Adults. J Spinal Disord Tech. 2008;21;477-483. [Crossref]

08. Labelle H, Mac-Thiong JM, Roussouly P. Spinopelvic sagittal balance of spondylolisthesis- a review and classification. Eur Spine $J$. 2011;20(Suppl 5)641-6.

[Crossref]

09. Mohammad A, Ahmad Y. Transforaminal Lumbar Interbody Fusion for the Treatment of Spondylolisthesis and Degenerative Segmental Instability, Surgical and Radiological Outcome. Egyptian Journal of Neurosurgery. 2015;30(3)213-220.

[Crossref]

10. Cheng $C L$, Fang D, Lee PC, Leong JC. Anterior spinal fusion for spondylolysis and isthmic spondylolisthesis, Long term results in adults. J bone Joint Surg Br. 1989;71;264-7.

[Crossref]
11. Debnath, Ujjwal $\mathrm{K}$, et al. Interbody Fusion in Low Grade Lumbar Spondylolsithesis- Clinical Outcome Does Not Correalte with Slip Reduction and Neural Foraminal Dimension. Asian Spine Journal. 2016;10(2)314-320.

[Crossref]

12. Gaetani $P$, Aimar E, Panella L, Levi D, Tancioni $F$, Di Ieva A, Debernardi A, Pisano P, Rodriguez y Baena R. Functional disability after instrumented stabilization in lumbar degenerative spondylolisthesis- a follow-up study. Funct Neurol. 2006;21(1)31-7.

[Crossref]

13. Pasha IF, Qureshi MA, Haider IZ, Malik AS, Qureshi MA, Bin Tahir U. Surgical treatment in lumbar spondylolisthesis- experience with 45 patients. J Ayub Med Coll Abbottabad. 2012;24(1)75-8.

[Crossref]

14. Park Y, Ha JW, Lee YT, Oh HC, Yoo JH, Kim HB. Surgical outcomes of minimal lyinvasive transforaminal lumbar interbody fusion for the treatment of spondylolisthesis and degenerative segmental instability. Asian Spine J. 2011;5(4)228-36.

[Crossref]

15. Liu ZD, Li XF, Qian L, Wu LM, Lao LF, Wang HT. Lever reduction using polyaxialscrew and rod fixation system for the treatment of degenerative lumbar spondylolisthesis with spinal stenosis- technique and clinical outcome. J Orthop Surg Res. $2015 ; 10 ; 29$.

[Crossref]

16. Kaftandziev I, Trpeski S, Filipce V, Arsovski O, Hasani I, Nikolov L, et al. Operative treatment of degenerative lumbar spine spondylolisthesis. PRILOZI. 2015;36(1)129-135.

[Crossref] 